\title{
Correction to: The effect of the information-motivation-behavioral skills (IMB) model variables on orthorexia nervosa behaviors of pregnant women
}

\author{
Ayşe Taştekin Ouyaba' ${ }^{1}$ · Pınar Çiçekoğlu Öztürk ${ }^{2}$ (D)
}

Published online: 7 July 2021

(c) Springer Nature Switzerland AG 2021

\section{Correction to: \\ Eating and Weight Disorders-Studies on Anorexia, \\ Bulimia and Obesity \\ https://doi.org/10.1007/s40519-021-01237-x}

Unfortunately, the Fig. 1 was incorrectly published in the original publication. The complete correct Fig. 1 is given below:

The original article can be found online at https://doi.org/10.1007/ s40519-021-01237-x.

Ayşe Taştekin Ouyaba

ayse.tastekin@hotmail.com

Pınar Çiçekoğlu Öztürk

pcicek78@hotmail.com

1 Obstetrics and Gynecology Nursing, Faculty of Health Sciences, Afyonkarahisar Health Sciences University, Afyonkarahisar, Turkey

2 Psychiatric Nursing, Fethiye Faculty of Health Sciences, Muğla S1tkı Kocman University, Muğla, Turkey 


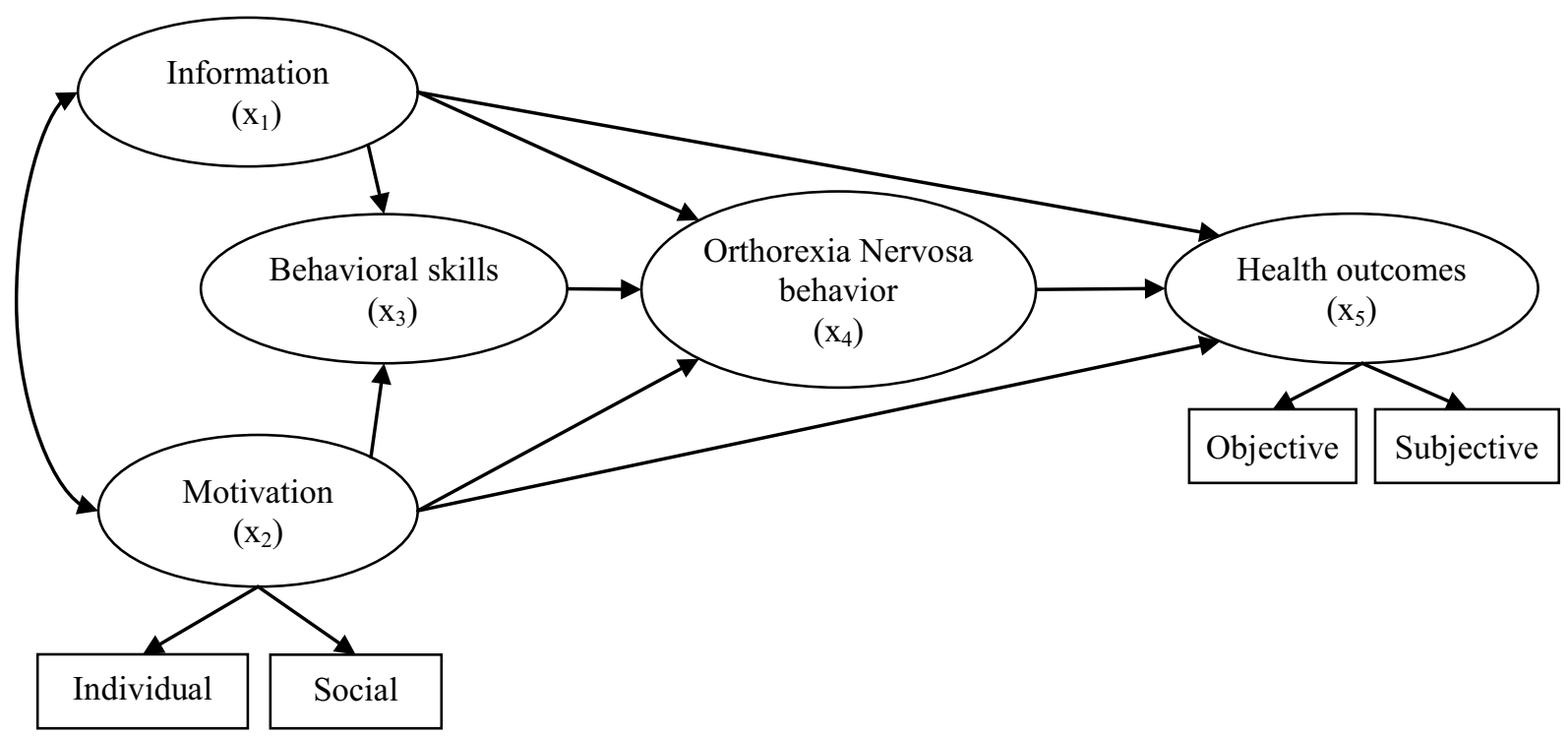

Fig. 1 Hypothetical model and research hypotheses: $\mathrm{H}_{1}\left(\mathrm{x}_{1} \rightarrow \mathrm{x}_{3}\right)$ Information affects behavioral skills. $\mathrm{H}_{2}\left(\mathrm{x}_{2} \rightarrow \mathrm{x}_{3}\right)$ motivation affects behavioral skills. $\mathrm{H}_{3}\left(\mathrm{x}_{1} \rightarrow \mathrm{x}_{4}\right)$ information affects Orthorexia Nervosa [ON] behaviors. $\mathrm{H}_{4}\left(\mathrm{x}_{2} \rightarrow \mathrm{x}_{4}\right)$ motivation affects $\mathrm{ON}$ behaviors.
$\mathrm{H}_{5}\left(\mathrm{x}_{3} \rightarrow \mathrm{x}_{4}\right)$ behavioral skills affect ON behaviors. $\mathrm{H}_{6}\left(\mathrm{x}_{1} \rightarrow \mathrm{x}_{5}\right)$ information affects health outcomes. $\mathrm{H}_{7}\left(\mathrm{x}_{2} \rightarrow \mathrm{x}_{5}\right)$ motivation affects health outcomes. $\mathrm{H}_{8}\left(\mathrm{x}_{4} \rightarrow \mathrm{x}_{5}\right)$ ON behaviors affect health outcomes

The original article has been corrected.

Publisher's Note Springer Nature remains neutral with regard to jurisdictional claims in published maps and institutional affiliations. 